\title{
Simulation Research on the Complexity of Life Game
}

\author{
Jiacheng Huang ${ }^{1}$ \\ ${ }^{1}$ College of Internet of Things Engineering, Jiangnan University, Wuxi, China \\ Correspondence: Jiacheng Huang, College of Internet of Things Engineering, Jiangnan University, Wuxi, China.
}

Received: October 5, 2021

Accepted: October 22, 2021

Online Published: October 26, 2021

doi:10.5539/cis.v14n4p65

URL: https://doi.org/10.5539/cis.v14n4p65

\begin{abstract}
The relationship between complexity and various factors is explored through the simulation of the three neighbor ways of the game of life. It mainly discusses the state evolution process of cell populations under various evolutionary laws, various environmental scales and various initial states. Based on the discovery of a novel, long-lived and simple cell with an initial state, the periodic and stable cell morphology in Game of Life is introduced, thus reflecting the related complexity factors and changes. By simulating various environmental boundaries and comparing the steady-state graphs, it is concluded that a closed system will cause certain limitations in the final outcome. The limited environment will prevent the cell from expanding outward, but it can also create more periodic patterns. A limited environment is simultaneously an important factor in simplifying the system. In addition to the environment, the edge of chaos is also an important factor in the complexity of the system. An appropriate evolution rule can help the entire system find a balance in the chaos and present stable and interesting patterns. In addition, the correct neighbor method has a positive effect on the change of the cell. Finally, an infinite loop mode is set up to illustrate once again the wonder and complexity of Game of Life.
\end{abstract}

Keywords: complexity, cell, state, life game

\section{Introduction to Cellular Automata}

Cellular automata is defined as a dynamic system which evolves in the discrete time dimension and a cell space composed of finite and discrete state cells in line with some system rules (Edwards \& Maignan, 2020).

It is a computational method based on computation or algorithm, which is used to compute and simulate natural phenomena in geometric space (Nandi et al, 1994). It is in a discrete state in time and space, each variable corresponds to a certain amount of states, and the rules for changing states are local in space and time, so its state changes can be regarded as synchronous data processing (Chopard \& Lagrava, 1999). Von Neumann, the father of computers, put forward relevant concepts to simulate the self-replication function of life systems (Jacobson, 1967). And Stephen Wolfram has done more in-depth research and exploration on the theory of cellular automata and complex systems in his book. He believes that the theory of cellular automata is a way for people to experiment and explore the nature of things in the computational universe (Aaronson, 2002). These experiments can be implemented through programs and codes (Mier \& Newman, 2020).

Macroscopic objects are continuously divided and refined, and they will become simpler and simpler, until they are finally abstracted out of quarks, electrons and other substances. For example, a car can be divided into many objects such as pedals, brakes, and steering wheels. The first objects are very complicated, but we continue to abstract things, and finally, we can get the simplest things. How to abstract and simplify in real life is a huge problem, but cellular automata is an effective method among them. Cellular automata based on the rule computation provides new ideas and methods for simulating natural phenomena and life phenomena. It has a wide range of applications, such as simulating urban growth (Couclelis, 1997), and traffic conditions (Zhao \& Xin, 2020). It can also be used in fields such as natural computing and life sciences (Guruprasad \& Sekar, 2006).

For example, Maxwell's equations are very interesting but very complex. It is unimaginable to model with such cumbersome equations, but we can continue to simplify it and replace it with the simplest algebraic rule. There are two reasons for the simplification. The first key point is that important terms need to be defined, such as current and voltage in the light bulb example. The same is true for cellular automata. Each cell can only have two states, defined as life or death. The second key point is to set a certain range. For example, in Maxwell's equations, we assume that the right side of the equation is 0 . This is a special state, and a complex system is 
composed of countless such states.

The divided grid is used to represent the cell space. There are three common neighbor methods: Von.Neumann type, moore-type and extended moore-type, as shown in Figure 1. The gray cell in the middle is the central cell, and the surrounding black cells are its neighbors. The state of the central cells at the next moment is related to their state at this time.

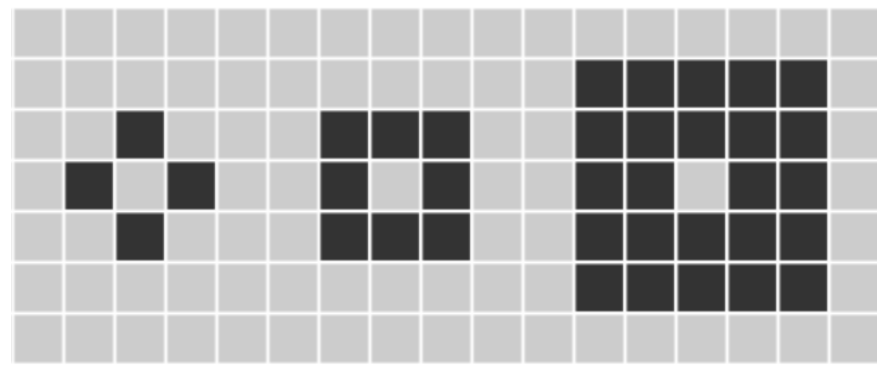

Figure 1. Three neighbor ways

At the beginning, the system has a certain number of cells with only one initial state, and then it evolves according to the rules. At this moment, each cell has its own state and position. When it comes to the next moment, all cells change according to their own state and uniform rules (Huang \& Peng, 2021). The whole process of change sounds simple, but the outcome is difficult to predict and analyze. Take a cellular automaton that can recognize contours as an example: this is a maple leaf-shaped cellular automaton. The rule is that if a cell is black and the adjacent cell is white, then this cell will remain Black, otherwise, the cell will become gray. Only by changing the adjacent cells, the entire graph has undergone a big change. This rule filters out the outline of the graph. The whole process is shown in Figure 2.

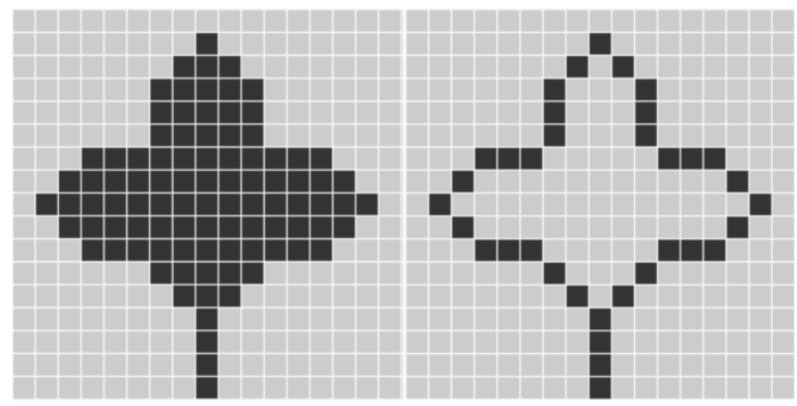

Figure 2. A cellular automata that recognizing external contours

The novelty of this article is to design a software that can simulate the game of life, which can intuitively simulate the changes of various cell groups. Through a simple group of cells with "longevity" properties that we independently discovered, the wonders of the game of life can be demonstrated, and various components after steady state can be observed. Setting up environments of various sizes and various initial states, it is observed that the environment and the edge of chaos are two important factors that affect the complexity of the system. Various initial states can achieve the same outcome under the influence of the environment. The edge of chaos mainly includes evolutionary rules and neighboring methods. The conclusion is reached through the controlled variable method: only a suitable balance point is found, the game of life will develop in a meaningful direction. At the same time, the symmetry of the initial state and the number of cells must be considered, and the evolution of the symmetric initial state is always symmetrically distributed (Huang \& Peng, 2021).

The rest of this paper is organized as follows, In Section 2, we introduced the related concepts and rules of the program. In Section 3, we introduced the design process and overall framework of the program. And in the fourth part, the results of the program and influencing factors are simulated and discussed.

\section{Introduction to Emergency and Life Game}

Among the commonalities of complex systems, there is one of the most striking universal phenomena-"emergence" (Cotsaftis, 2009). Emergence means that individuals in the system follow simple rules. 
When a whole is formed through local interactions, some new attributes or rules will suddenly be born at the system level. This phenomenon is emergence. Therefore, emergent attributes or laws do not break individual rules, but it cannot be simply explained by low-level individuals. So, to put it simply, emergence can be understood as "the whole of the system is greater than the sum of its parts" (Hsien et al, 1998).

Conway's Game of Life is the most famous "emerging" program in the computer field. In 1970s, Conway compiled this game program after a lot of experiments (Adamatzky, 2010). The rules of the whole game are very clear and simple, but it quickly gained popularity and attention all over the world (Gadi et al, 2014). To a certain extent, it reflects the laws of survival and evolution of life in nature. (Lorena \& Sergio, 2016).

The entire interface is represented by two-color squares, and each cell occupies a square. In addition, each cell has two states: "alive" or "dead" (Packard \& Wolfram, 1985). Black squares represent living cells, and gray squares represent dead cells. There is a circle of 8 cells around each cell. The state of the cell changes according to the following rules:

1. Survival: If a living cell (black) wants to survive (keep black), it needs at least 2 or 3 living cells around it;

2. Death: If the number of living cells around a cell (black) is more than 3 or less than 2, it will die (black becomes gray);

3. Resurrection: If there are exactly 3 living cells around a dead cell (gray), the cell will be resurrected (gray to black).

There is always a state of balance in nature. At the same time, the goal of human beings' constant desire and exploration has always been to find balance in the chaos. In real life, situations similar to game of life also exist, such as the daily entertainment activities in China: mahjong and poker. The concept of mahjong is to create order out of chaos based on random drawing of titles.

\section{Design of the Software}

Three neighbor modes and the size of the environment are set up. Taking the moore-type neighbor method as an example, first count the number of live cells in the valid range. Then change the state of the cell according to the rules, Finally, assign the generated state to the current state, and then enter the next change:

There are three small menus the simulation interface of the software. The "Options" menu is used to select the neighbor ways and operating time, as shown in Figure 3. Although there are three neighbor modes, the only one that makes sense is the moore-type. After selecting an initial state, we can choose "Start", and the system will operate according to the rules. Throughout the process of change, we can choose "Stop" to observe the current cell state, or choose "Start" to restart the game.

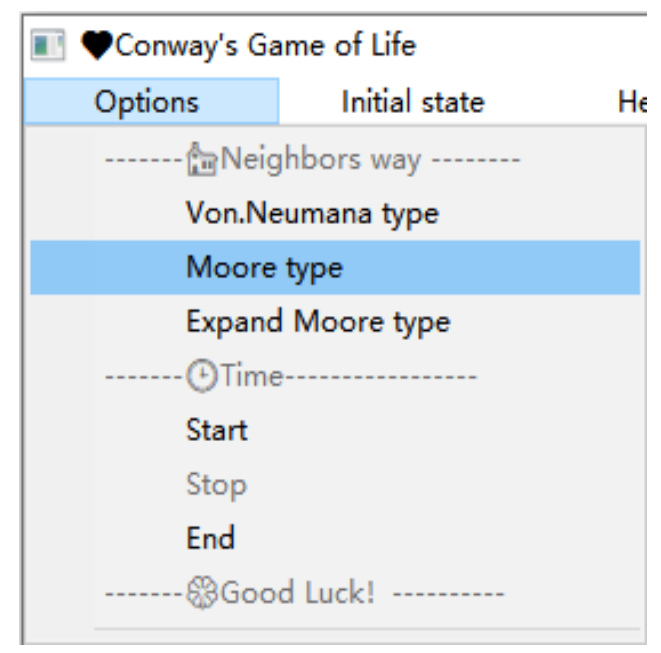

Figure 3. "Options" menu interface

The function of the "Initial State" menu is to set the initial state of the cell, and there are three setting methods, as shown in Figure 4. The first way is code setting, and we can initialize the state of a large number of cells by this way. The second way is manual setting, which can be used to find some meaningful patterns. The third type is random setting, which can be used to simulate some special situations. A total of four sizes can be selected. 
The first three cases are relatively stable.

\begin{tabular}{|c|}
\hline Initial state Help \\
\hline--- State setting----- \\
Code setting \\
Manual setting \\
$10 \times 10$ \\
$30 \times 30$ \\
$50 \times 50$ \\
Huge size \\
- Environment----- \\
Little random \\
Big random
\end{tabular}

Figure 4. "Initial state" menu

The "Help" menu briefly introduces the relevant information of the software, as shown in Figure 5.

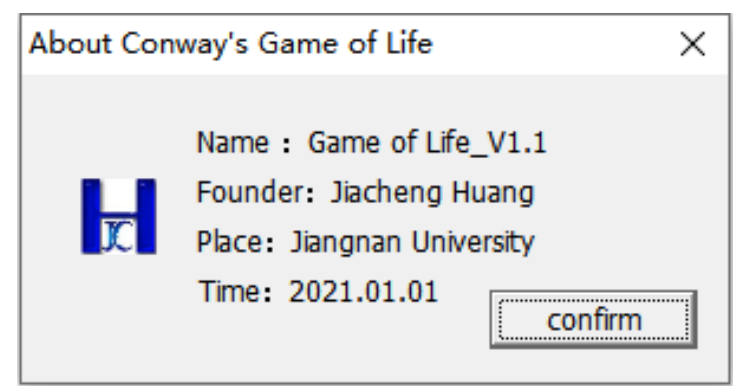

Figure 5. "Help" menu

When we use the software, first select the neighbor mode, and then the initial state. In the game, we can choose to restart the game or exit the game, otherwise, it will continue until the state is stable.

\section{Graphical Simulation and Outcome Analysis}

Since the appearance of Game of Life, it has triggered extensive discussions and explorations among fans all over the world. Here, we list a simple and long-lived graph discovered by me to show its change process and introduce various graphs after steady state. The whole graph has nine cells, as shown in Figure 6.

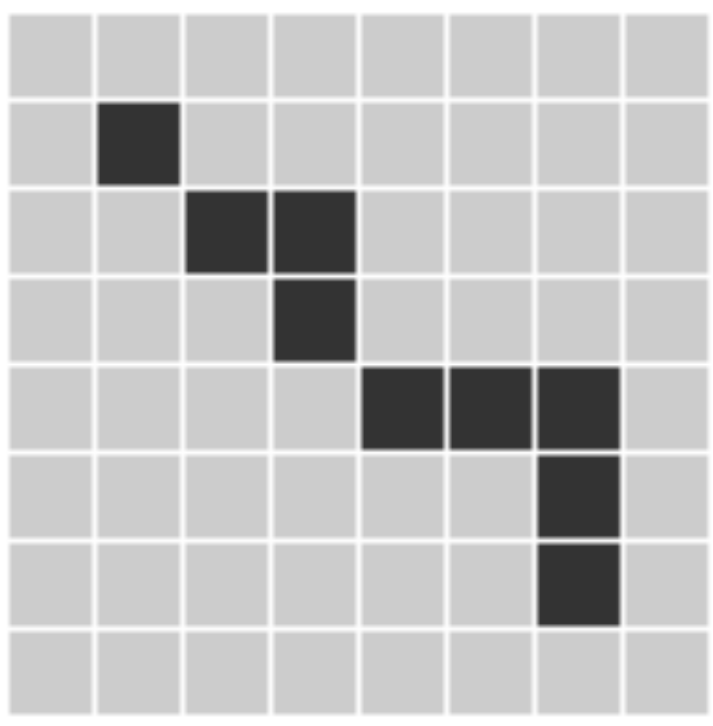

Figure 6. A simple, long-lived initial state 
It takes a long time to stabilize after as many as 2476 changes. There will be a process of simplification-complexity-simplification during this time. The central area of the final state is presented in Figure 7.

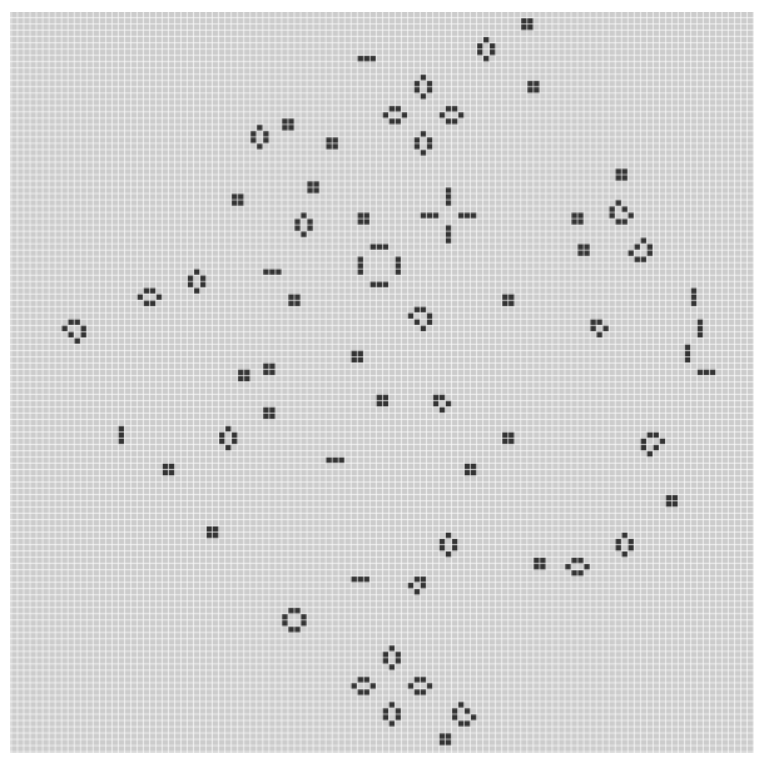

Figure 7. Central area of the final state

The entire central area consists of two small parts: periodic type and fixed type. Four types of fixed types appear here, as shown in Figure 8. The fixed cell groups mean that when the system is stable, they will not change any more. It is as if a dandelion seed is continuously spread by the wind, and will eventually become a sea of flowers. But the entire process of diffusion is unpredictable.
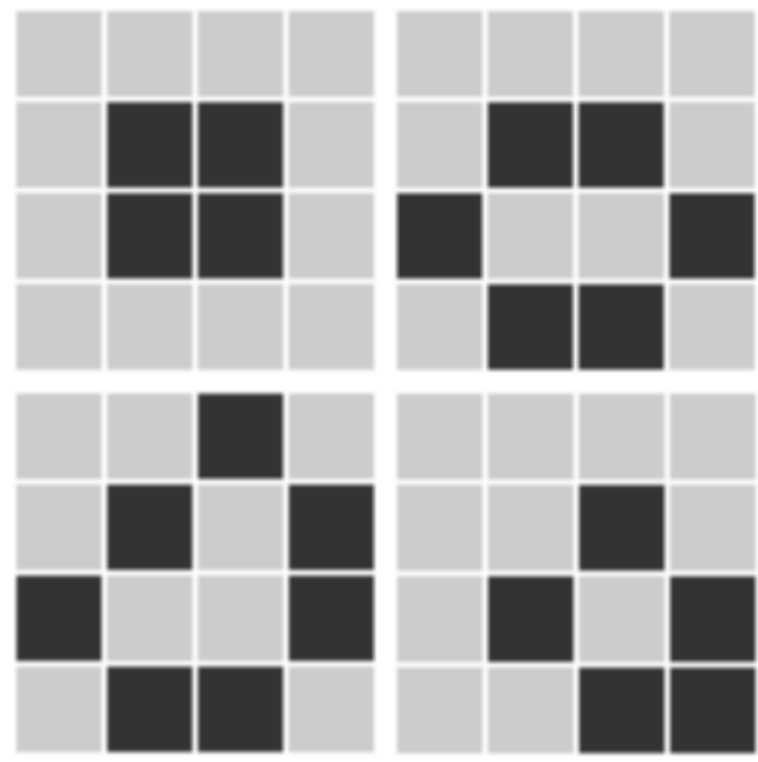

Figure 8. Four stable "fixed" cell groups

There are "gliders" emanated by from the central area around the graph (Bell, 1993). In the game of life, "gliders" are special graphs with a period of 4 and can move continuously along a certain track, as shown in Figure 9. 


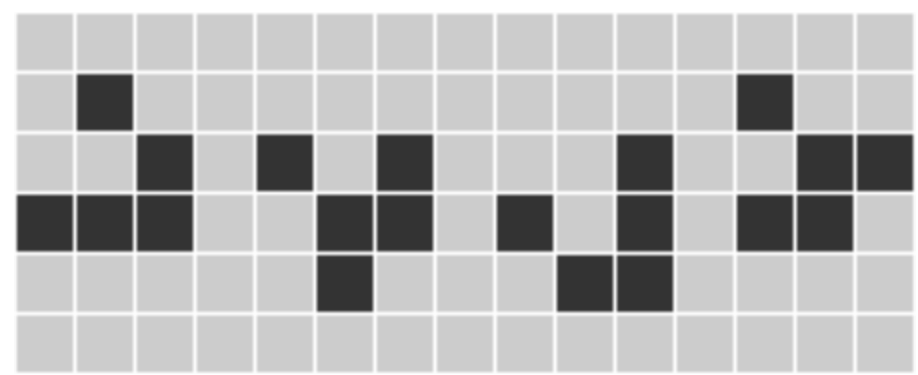

Figure 9. Cell group similar to a glider

\subsection{The impact of environment size on system complexity}

First, select the size of the environment of $10 \times 10$, adopt the moore- type neighbor method, and set an initial state. At this time, the boundary of the system is closed, and it can be found that it has a total of 5 states, as shown in Figure 10. The whole process continues to circulate inside and outside.
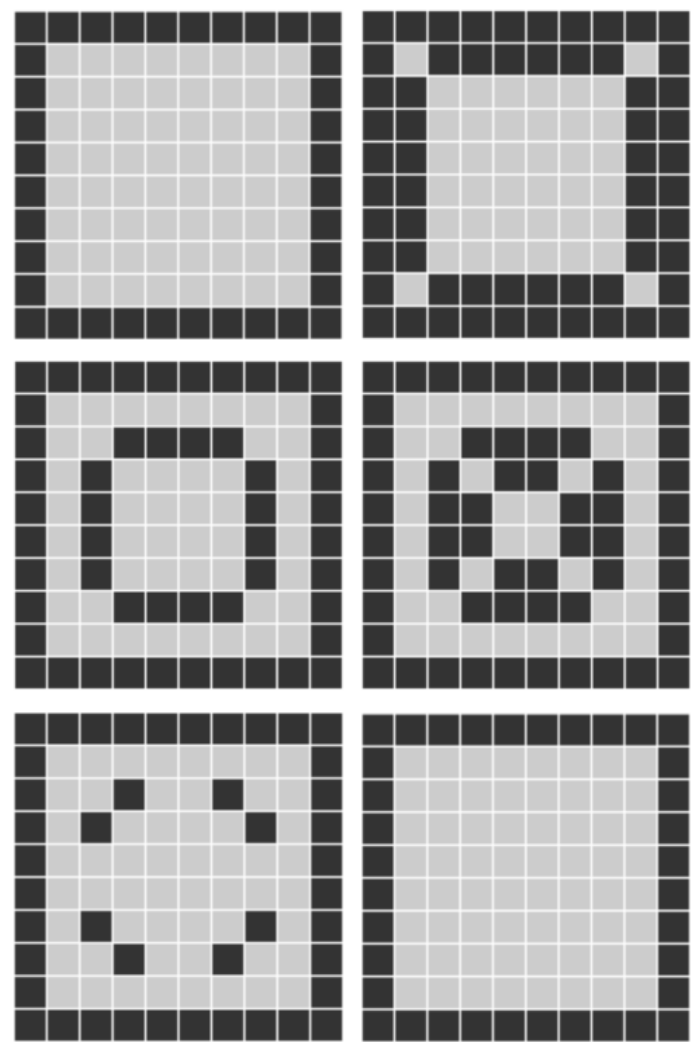

Figure 10. A cell group with a period of five

If the size of the environment is enlarged, it will be found that a stable state is formed after 10 changes. As shown in Figure 11, it can be seen that the closure of the environment affects the change of the graph, which prevents the cell from expanding outward. 

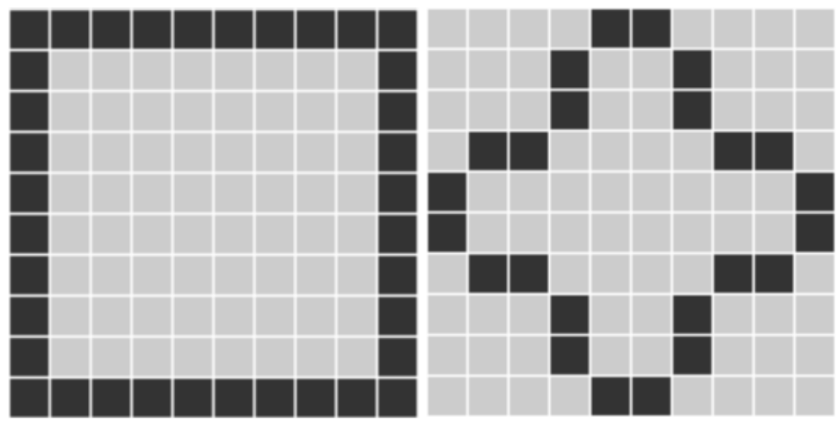

Figure 11. The process of change in an infinite environment

The size of the initial state and the environment are expanded at the same time, and the same situation exists. Set up a shape similar to a square with a side length of 26, as shown in Figure 12. Then set various border sizes and observe the final outcome. The first case has a boundary of 30 and is affected by the environment, and the second case has a larger boundary and is not affected by the environment. The comparison between the two graphics is represented in Figure 13.

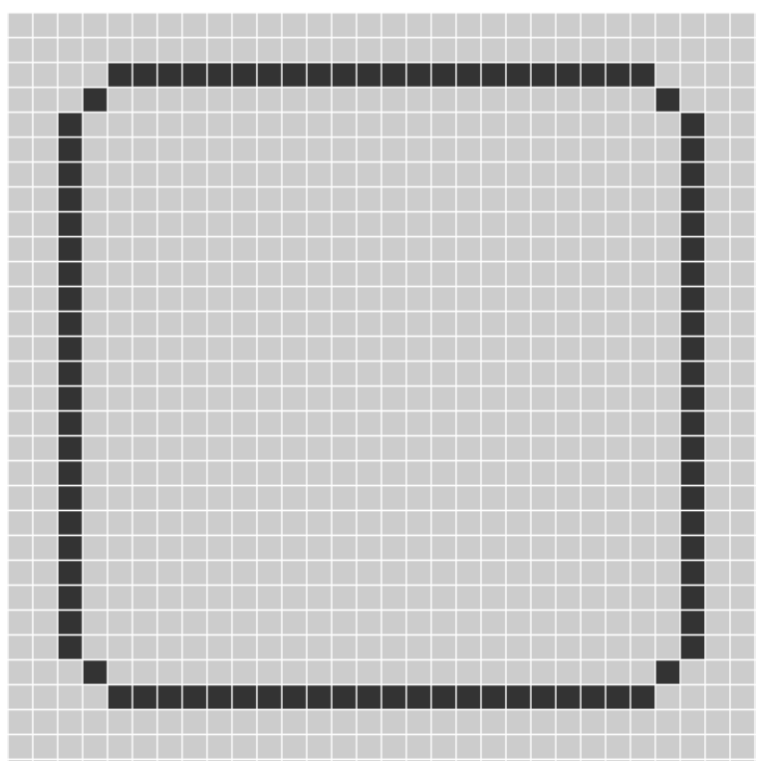

Figure 12. "Square" with a side length of 26
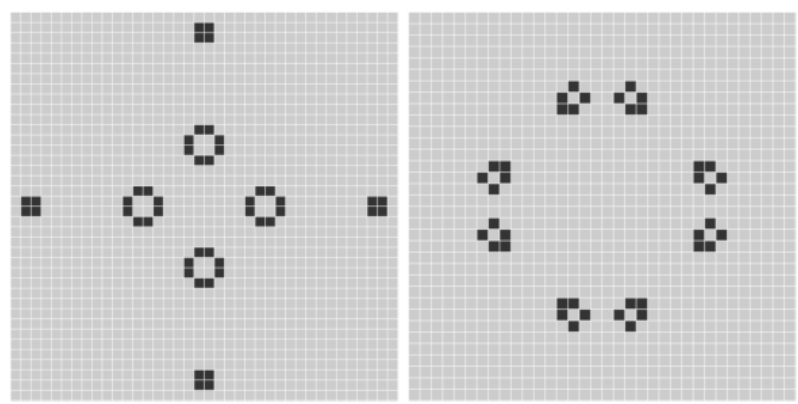

Figure 13.Final states without or with boundary influence

Then, select squares with side lengths of 46 and 42 and proceed without environmental restrictions. The entire process is shown in Figure 14 and Figure 15, respectively. 


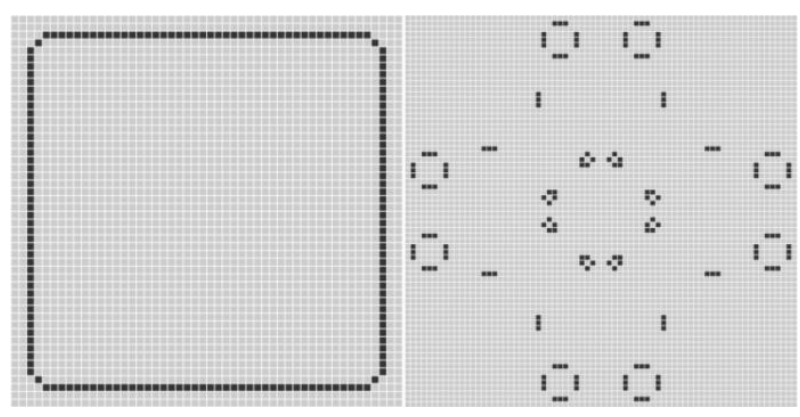

Figure 14. The initial and final state of a square with a side length of 46

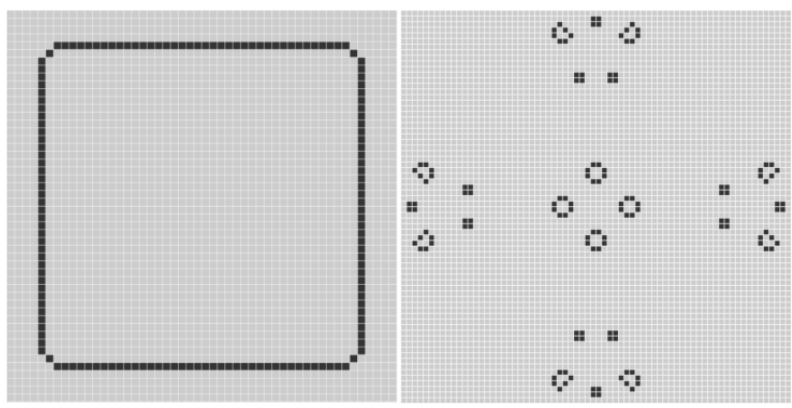

Figure 15 . The initial and final state of a square with a side length of 42

When the environment boundary is 50 in the above two cases, the cell collides with the boundary during the diffusion process, and finally forms an identical figure, as shown in Figure 16. It can be clearly seen from these examples that the changes of cells are affected by the environment. When the environment is restricted, the cell will merge and collide with the boundary in the process of continuous expansion, thus changing inward again.

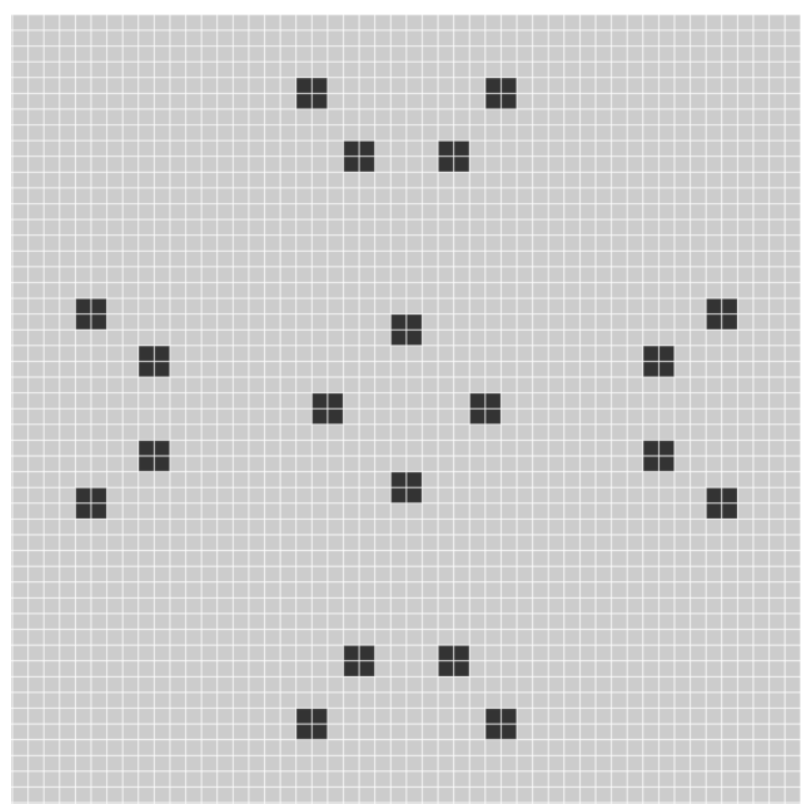

Figure 16. The same final state with boundary constraints

In the system, the same destination can be reached by multiple paths. We can try to get the same final state more easily by changing the initial state of the cell group (Cotsaftis, 2009). Although currently in a limited environment, the number of cells has not changed much. But when the size of the enclosed environment 
becomes larger, the variety of choices can greatly simplify the process of the system and improve efficiency.

For example, as shown in Figure 17, one represents a flower and the other is a graffiti. A flower can reach a steady state after 5 changes, while graffiti needs up to 73 changes to reach a steady state.

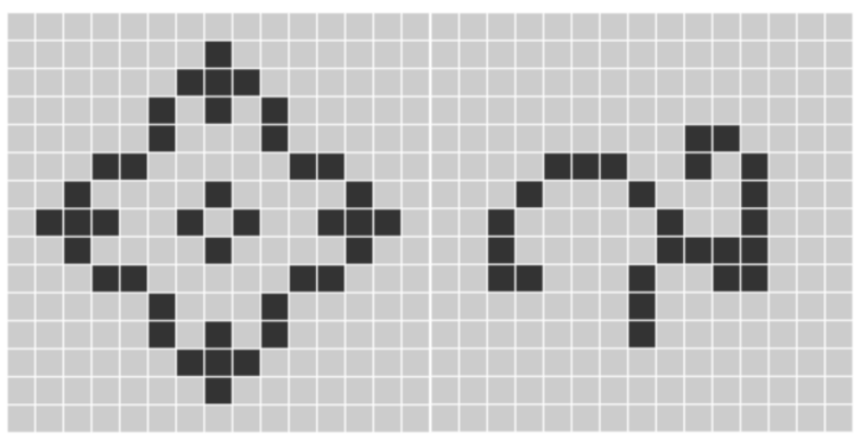

Figure 17. The flower and doodle

Interestingly, the results of both changes are the same, as shown in Figure 18. It can be seen more intuitively that a suitable initial state can greatly simplify the evolution process. At the same time, the closed nature of the environment will greatly increase the complexity of the system, which will be described in more detail below.

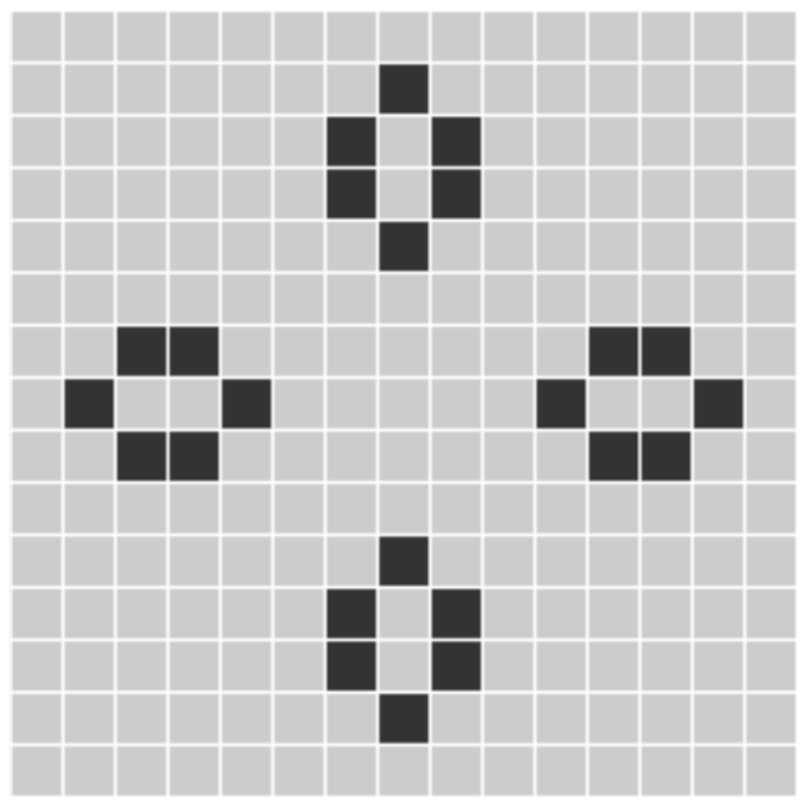

Figure 18. The same final state

\subsection{The Influence of the Edge of Chaos on System Complexity}

The edge of chaos is related to two factors here, one is neighbor mode and the other is cell evolution rule. According to scientific theory, the individual rules in all emerging systems are on the edge of chaos and order. If we regard the power of chaos as a tension that destroys the system and the power of order as a cohesive force that prevents destruction and forms an orderly structure, then only when the two forces struggle and balance each other can the system emerge and create an endless stream of complex structures.

The introduction of random variables into the system can make the simulation outcome closer to the actual situation. Set up three neighbor methods and initialize them randomly. The initial state is shown in Figure 19. 


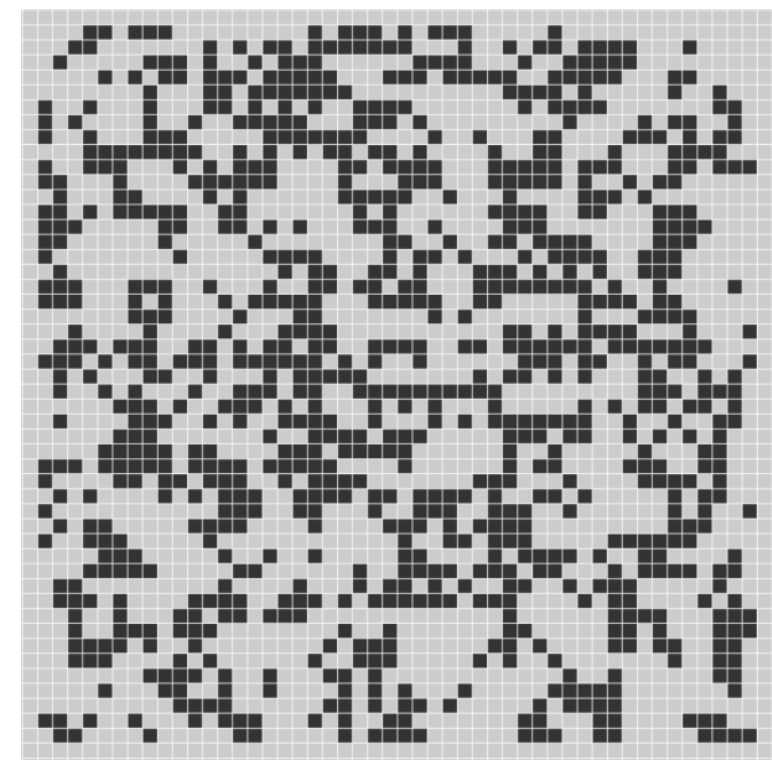

Figure 19. The initial state of randomization

The Von. Neumann-type can reach a stable state after only 8 changes. The change process is too simple and obviously cannot be used as a main neighbor way, as shown in Figure 20.

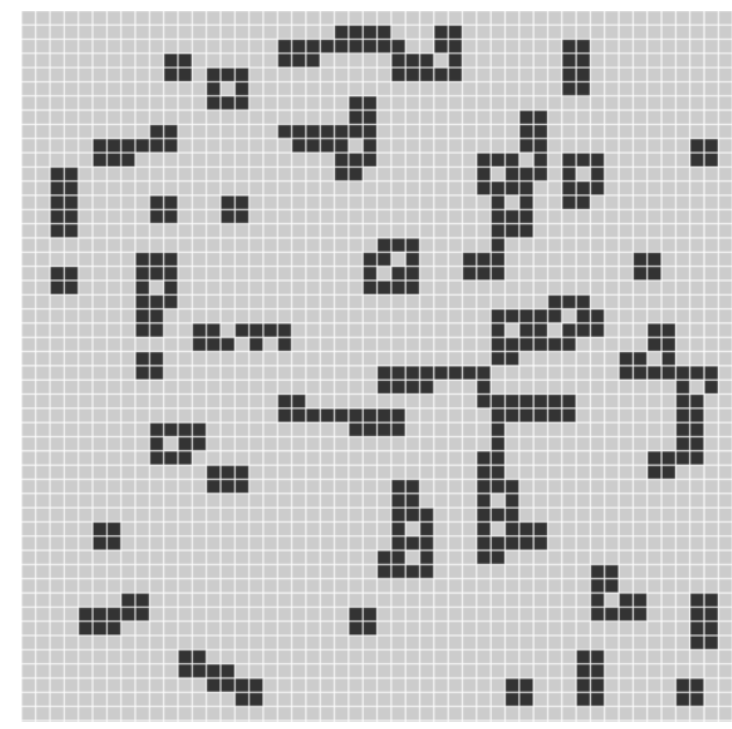

Figure 20. The final state of von Neumann type

The moore-type changes 540 times under the same initial state, which is more than 60 times that of the von type, before reaching a stable state, as shown in Figure 21. Because of the limitations of a closed system, individual cells on the edge can also survive. In order to reduce the impact of the boundary, we use the outermost layer as an insulating layer. We think the limited environment is also an important influencing factor, which leads to the complexity of the system, similar to the splashing of water droplets. When the cell group encounters the cells at the edge of the system, more splashes will be emitted and become more complicated. When the entire system is infinite, the moore-type cell group will continue to expand and multiply, but eventually, it will become very simple. 


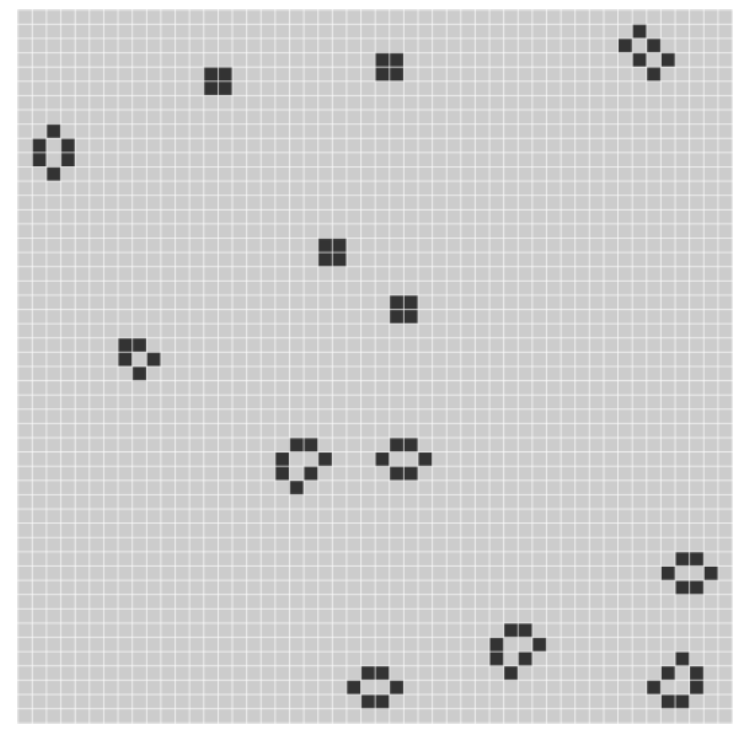

Figure 21. The final state of moore-type

However, the expanded moore-type is more complicated, and the change process is always in a chaotic situation. It can be seen from this that an appropriate neighbor mode can make the system reach balance, thus resulting in a relatively orderly and complex structure.

The second important factor is the evolution rule. The cell rule in the game of life is that if there are three living cells among the eight adjacent cells of a dead cell, the cell can be resurrected. This means that if there are three black squares, a new black square can be generated. Respectively, change the rules too if there are 7 or 1 black grids in the neighbors, a new black grid will be generated. Observe the generated graphics and find that the final situation will soon be in a quiet situation or too chaotic. The meaningful patterns are no longer produced, and interesting graphics will no longer appear. First, set a simple initial state randomly. When a black square can produce a new black square, after only a few changes, the entire graph becomes extremely chaotic, and no meaningful patterns can be found. The whole process is shown in Figure 22. In the same initial state, when the rule is set to 7 black squares, the entire page will be cleared after only three changes.

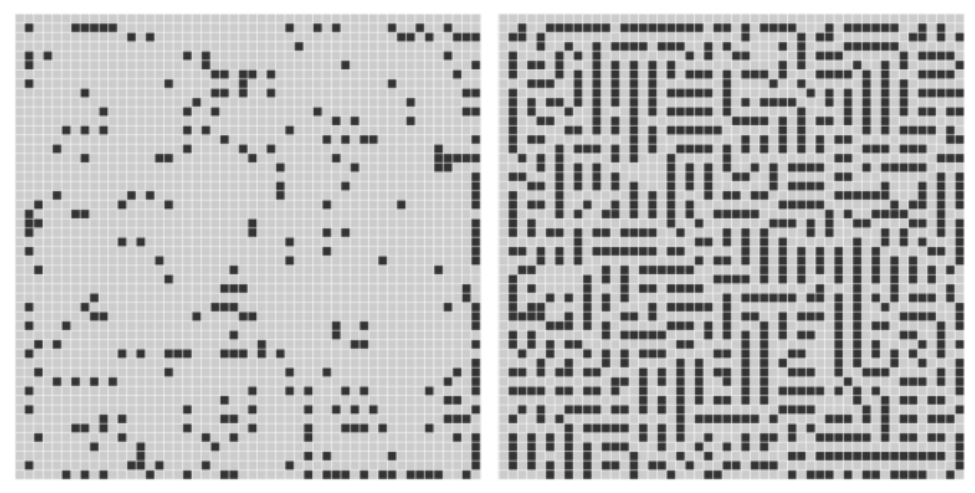

Figure 22. Another rule of evolution

Therefore, the edge of chaos is very important for complex systems. For Game of Life, an appropriate neighbor mode and evolution rules can better represent the process of life evolution, making the system more complex and interesting. A proper balance point will make the whole game develop in a more meaningful direction. Cellular automata follow simple rules, and the mathematical formulas or codes used to calculate them are very simple, but this is also the most important. The use of cellular automata must not exceed the boundaries of the rules, otherwise, it will cause trouble and fall into chaos. Similarly, the game of life must follow certain evolutionary rules, in order to present interesting patterns. 


\subsection{Other Factors Related to System Complexity}

First, the space division here is in the form of a square grid. Compared with the triangular and hexagonal division methods, the square grid has the advantages of intuitiveness and simplicity, and is particularly suitable for expression and display in the existing computer environment. However, it cannot simulate isotropic phenomena well. In most cases, the final stable state of the system is some symmetrical structure. The symmetrical structure is easier to survive in the system. At the same time, it can be concluded from the changes of various squares above that the initial state of symmetry is always symmetrically distributed. When the initial state is asymmetrical, the graph may transform into a symmetrical structure at a certain moment, and finally, become a symmetrical distribution state. As shown in Figure 23, the disordered initial state becomes symmetrical after 13 changes, and after 18 changes becoming steady.

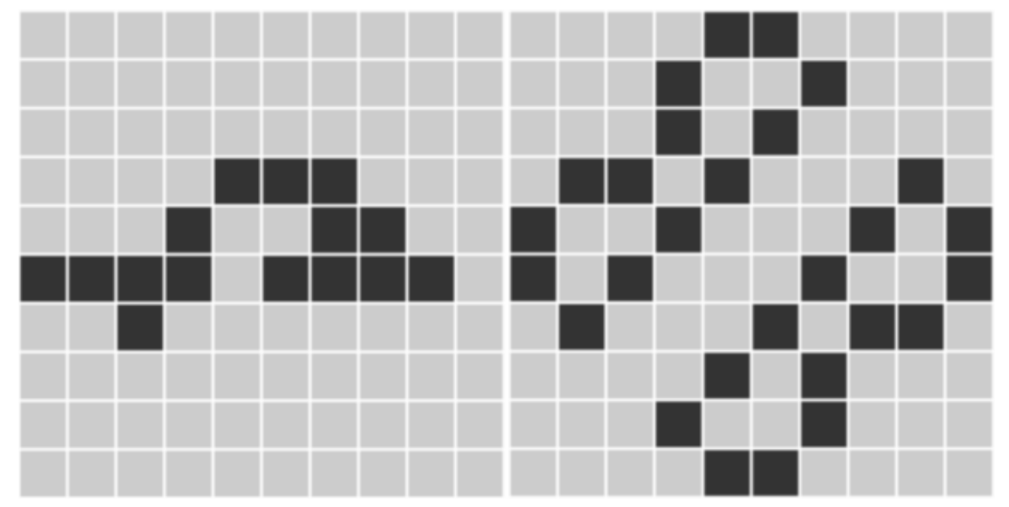

Figure 23. Asymmetry changes into symmetry

Secondly, We think the game of life is related to the non-linear interaction of a large number of individuals. If the size of the environment in the game of life is limited to 10, it is difficult to observe a simple structure such as a "glider". The reason is that as soon as it is produced, it will be submerged in other structures. If the environment is large enough, but the number of cells is small, some interesting structures cannot be observed. The true game of life requires a large number of individuals to play in an infinite environment.

The outcome of Life Game is difficult to be predicted. However, the artificial use of rules can also create an infinite loop pattern. We set up a scene where we can keep moving, as shown in Figure 24. 10 gliders will move counterclockwise periodically in the central area, and four spacecraft will move back and forth on the space station around the graph. The spacecraft will show periodic changes and will continuously supply and distribute supplies. The combination of the spacecraft and the capsule can exist and operate independently. The part in the upper left corner explains that when the spacecraft sends a supply to the approaching glider behind it, the glider changes its posture to receive the supplies and then changes into a new glider shape, thus changing its trajectory and continuing to fly, as shown in Figure 25. 


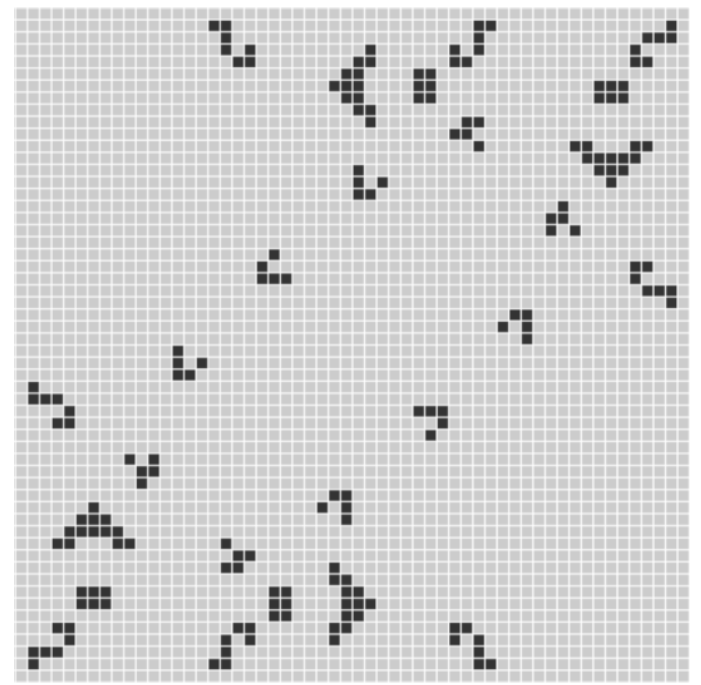

Figure 24. An endless loop mode

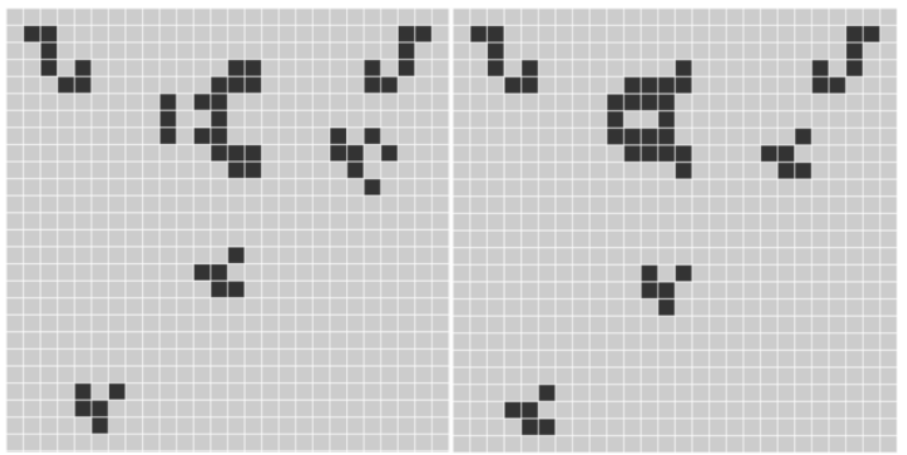

Figure 25. A new "glider" is produced

\section{Conclusion}

Visual studio2010 is used as a simulation tool to complete the entire design. Through a simple, long-lived initial cell we discovered, the complexity and interest of the game of life are fully demonstrated. The controlled variable method is adopted to separately explore the influence of the size of the environment, the way of neighbors, and the evolutionary rules on the game of life. The simulation outcome shows that the game of life is complicated. The closedness of the limited environment will increase the complexity of the system and affect the final layout of the cell group. At the same time, there are multiple situations that can achieve the same outcome, which provides more possibilities for simplifying the system. Neighbor methods and evolution rules are very important to the complexity of the system. Finding a balance point is of great help to our research on complex systems. The game of life provides a new way of thinking for solving complex scientific problems. The microscopic individuals who follow simple logic show complex phenomena at the macro level. We think this is why economics distinguishes between microeconomics and macroeconomics.

The game of life is just a computer simulation, which is various from real life or ideal state. Just like a battery in an ideal state, the current tends to infinity when the voltage is increased. In the game of life, when the environment or other factors tend to be infinite, the cells will continue to multiply in all directions to create more rich and interesting graphics. But in reality, the battery will turn into ashes with a beam of flame, and the cells will gradually stabilize in the chaos. This is unpredictable in abstraction, and it is also a breakthrough for computer simulation in the future.

\section{References}

Aaronson, S. (2002). Book review: 'a new kind of science'. Physics, 56(2), 410-423. https://doi.org/10.26421/QIC2.5-7 
Adamatzky, A. (2010). Game of Life Cellular Automata. Springer. https://doi.org/10.1007/978-1-84996-217-9

Bell, D. I. (1993). Spaceships in conway's game of life. URL: http://research. germany. eu. net, 8(3), 601-604.

Chopard, B., \& Lagrava, D (1999). Book review: cellular automata modeling of physical systems. Journal of Statistical Physics, 97(5/6), 1031-1032.

Cotsaftis, M. (2009). What Makes a System Complex? an Approach to Self-Organization and Emergence. Springer Berlin Heidelberg. https://doi.org/10.1007/978-3-642-02199-2_3

Couclelis, H. (1997). From cellular automata to urban models: new principles for model development and implementation. Environment \& Planning B Planning \& Design. https://doi.org/10.1068/b240165

Edwards, R., \& Maignan, A. (2020). A class of discrete dynamical systems with properties of both cellular automata and 1-systems. Natural Computing, 19(3). https://doi.org/10.1007/s11047-019-09739-5

Gadi, O., Shlomo, W. Y., \& Be'ery. (2014). Computational methods for conway's game of life cellular automaton. Journal of Computational Science.

Guruprasad, S., \& Sekar, K. (2006). Artificial life and living systems: insight into artificial life and its implications in life science research. Bioinformation, l(4). https://doi.org/10.6026/97320630001139

Huang, J., \& Peng, Y. (2021). Simulation of life game based on cellular automata. Journal of Computer and Communications, 09(1), 44-58. https://doi.org/10.4236/jcc.2021.91005

Hui-Hsien, C., James, A., \& Reggia. (1997). Emergence of self-replicating structures in a cellular automata space. Physica D: Nonlinear Phenomena.

Jacobson, H. (1967). Book review:theory of self-reproducing automata. john von neumann, arthur w. burks. Quarterly Review of Biology, 42(4). https://doi.org/10.1086/405504

Lorena, C., Bob, H., \& Sergio, H. (2016). Conway's "game of life" and the epigenetic principle. Frontiers in Cellular \& Infection Microbiology, 6. https://doi.org/10.3389/fcimb.2016.00057

Mier, J. A., R Sánchez, \& Newman, D. E. (2020). Tracer particle transport dynamics in the diffusive sandpile cellular automaton. Chaos Solitons \& Fractals, 140(3), 110117. https://doi.org/10.1016/j.chaos.2020.110117

Nandi, S., Kar, B., K., \& Chaudhuri, P. P. (1994). Theory and applications of cellular automata in cryptography. Computers, IEEE Transactions on. https://doi.org/10.1109/12.338094

Packard, N. H., \& Wolfram, S. (1985). Two-dimensional cellular automata. Journal of Statistical Physics, 38(5), 901-946. https://doi.org/10.1007/BF01010423

Zhao, H. T., Zhao, X., \& Xin, L. Y. (2020). Cellular automaton model for three-lane urban road considering internet of vehicles lane. International Journal of Modern Physics C. https://doi.org/10.1142/S012918312050179X

\section{Copyrights}

Copyright for this article is retained by the author(s), with first publication rights granted to the journal.

This is an open-access article distributed under the terms and conditions of the Creative Commons Attribution license (http://creativecommons.org/licenses/by/4.0/). 